\title{
Evaluating heat stress tolerance and molecular relationship among inbred lines of maize during early generations
}

\author{
Anwer, A.M. ${ }^{1}$, Kh.M. Ibrahim ${ }^{2}$ and T. Bashandy ${ }^{1, *}$ \\ ${ }^{I}$ Genetics Department, Faculty of Agriculture, New Valley University, New Valley, Egypt. \\ ${ }^{2}$ Agronomy Department, Faculty of Agriculture, New Valley University, New Valley, Egypt.
}

\begin{abstract}
Heat stress is one of abiotic stress that has deleterious effects on crops yield. Therefore, this study aims are to evaluate heat-tolerant maize lines and characterizing them by DNA molecular markers. Inbred lines were generated for two generations by selfing pollination. They were evaluated in each generation for the performance of some agro-morphological traits under normal and heat stress conditions. All the traits of the $S_{1}$ and $S_{2}$ inbred lines varied significantly among them under both conditions, except plant height was not significant under heat stress for the $\mathrm{S}_{1}$ lines. The L6 inbred line had the highest yield under the stress conditions in both $S_{1}$ and $S_{2}$ generations. Moreover, the heat susceptible index showed that the lines, L6 and L40 were the highest tolerant in the both generations. Furthermore, cluster analysis based on morphological traits for the 5 selected $S_{2}$ inbred lines could be able to isolate the worst $S_{2}$ inbred line under heat stress conditions in an independent cluster. In addition, they were characterized by ISSR and SRAP molecular markers. The ISSR detected higher polymorphism (79.79\%) than SRAP marker (58.46\%). The ISSR clustering patterns managed to classify the highest yield line (L6) under the heat stress in a separated cluster, but both the SRAP and combined isolated the worst line (L32) in one cluster. The Mantel's test showed a positive correlation among all the studied markers. Additionally, the correlation was significant and highly strong $(\mathrm{r}=0.915)$ between morphological traits under normal conditions and SRAP marker. However, the identified $\mathrm{S}_{2}$ inbred lines with resistance to heat tolerance could be a beneficial source in the development of heat-tolerant maize hybrids.
\end{abstract}

Keywords: HSI; ISSR; maize; molecular markers; SRAP.

\section{Introduction}

Maize (Zea mays L.) is one of the most important cereal crops all over the world. It plays one of the main suppliers of food, feed, fuel, and fodder for millions of poor farmers, also in animal feed, and many industrial targets (Osti, 2019). The world production is about 967 million tons; about $35 \%$ of them are produced only by the United States of America (USA). In Egypt, the cultivated area is around 994818 ha with an annual production of 7450000 tones (FAO, 2019). Heat stress is one of several abiotic stress factors that affect worldwide maize productivity (Rowhani et al., 2011). Whereas, the exposure of maize for heat stress $\left(>30^{\circ} \mathrm{C}\right)$ for an extended period the grain output drops dramatically, while $20-22{ }^{\circ} \mathrm{C}$ is the average ideal temperature for the entire growth season (Schauberger et al., 2017).

\footnotetext{
*Corresponding author: T. Bashandy,

Email: bashandy@agr.nvu.edu.eg

Received: August 6, 2021; Accepted: August 19, 2021;

Available online : August 19, 2021.

(C) Published by South Valley University.

This is an open access article licensed under @c)(이)
}

Generally, temperature fluctuations disrupt the photosynthetic process, damage biological membranes, reduce nutrient uptake, and limit the action of numerous enzymes. Moreover, its effect during the reproductive period that produces dried silks, low seed germination and sterility of pollens resulting in a sever drop in yield (Sánchez et al., 2014). To face the heat damaging effects and the everincreasing demand, must improve genotypes that have both high yield and heat tolerance characteristics. Moreover, increasing maize production is one of the major efficient approaches for food security in developing countries. DNA molecular markers are the accurate techniques for the determination of genetic diversity among different genotypes. They are preferable than morphological characterization in that they are not affected by environmental changes. In addition, they are screening along with all genomic sequences of the organism (Prasad et al., 2009). Each of Inter Simple Sequence Repeat (ISSR) and Sequence Related Amplified Polymorphism (SRAP) markers are 
dominant PCR based molecular markers and have widely been applied in plants (Liu et al., 2008; Shao et al., 2010; Liu et al., 2012; Bashandy and ElShaieny, 2016; Luo et al., 2020; Mahmoud and Abd El-Fatah, 2020). The ISSR uses two simple sequence repeat to amplify the DNA fragments inter them without the need for any prior sequence information (Zietkiewicz et al., 1994). On the other hand, the SRAP marker technique amplifies the coding regions or Open Reading Frames (ORFs). Its action is based on using a forward primer to amplify the exon sequences and reverse primer specific to the intron and promoter regions ( $\mathrm{Li}$ and Quiros, 2001).The present study is aimed to develop heat-tolerant maize inbred lines and identify them by DNA molecular markers.

\section{Materials and Methods}

\subsection{Plant materials and field experiment}

The field experiment was carried out at the experimental farm of Agriculture Faculty, New Valley University, Egypt for three years 2017, 2018 and 2019. In the first year, $S_{1}$ generation was generated by the cultivation of one Maize population
Corn Belt Composite E.T.O (was provided by the National Maize Research program) on the $14^{\text {th }}$ of March. Then, 200 strong and healthy plants were chosen before silking, and were self-pollinated. After the harvest, 60 selfed ears ( $S_{1}$ seeds) that had enough seeds were selected and were individually shelled. In the second year, the $S_{1}$ generation was evaluated for the heat stress by growing the $60 \mathrm{~S}_{1}$ inbred lines at the two planting dates, 15 March 2018 as the heat stress time and 15 July 2018 as the normal planting time. The date of the normal planting time was chosen to avoid excessive heat during the pollination and grainfilling period. Furthermore, selfing pollination was performed for each $S_{1}$ inbred line to produce the $S_{2}$ seeds. In the third year, the $S_{2}$ seeds of the most tolerant $\mathrm{S}_{1}$ lines (29 lines that had HSI value less than 1.00) and the worst line (L32, as a negative control) were selected and grown under the same conditions as the $S_{1}$ inbred lines cultivation. The experimental layout was a Randomized Complete Block Design with two replicates. Each plot consisted of one row $0.70 \mathrm{~m}$ apart, three meters in length and 13 hills. The weather conditions of the cultivation seasons are shown in Fig. 1.

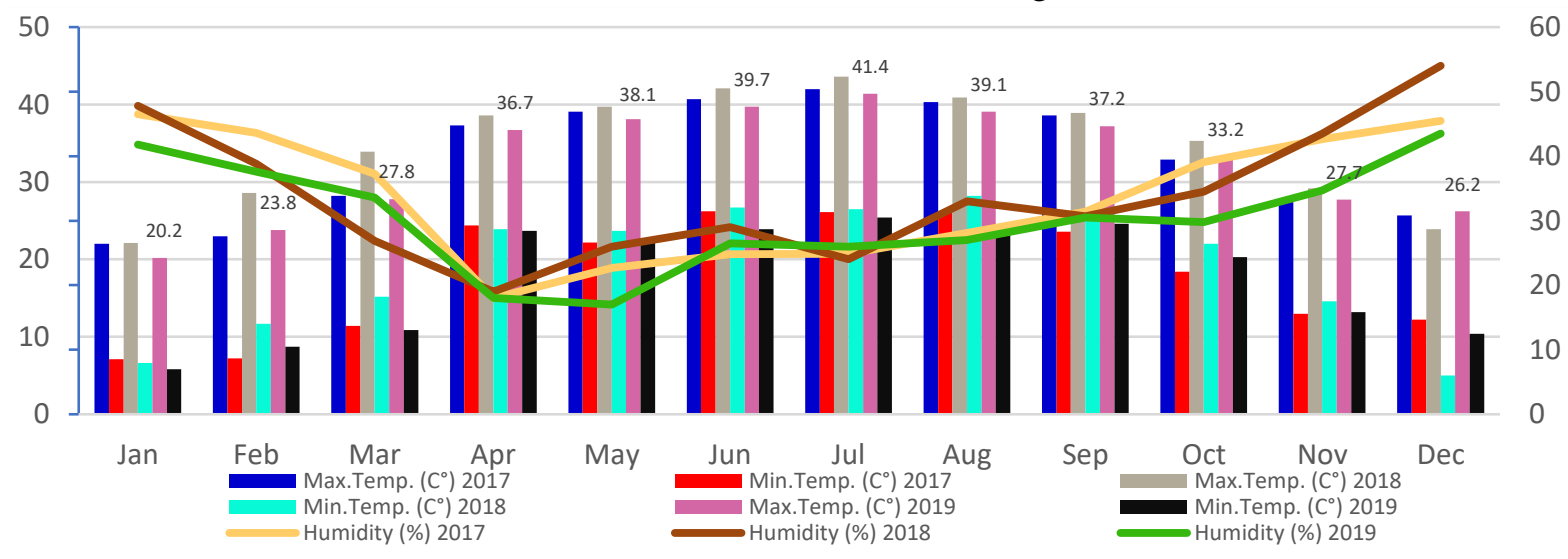

Fig. 1. Weather data of research farm during 2017, 2018 and 2019 (Meteorological station, El-Kharga, New Valley, Egypt).

\subsection{Traits measured}

Both the $S_{1}$ and $S_{2}$ generations were evaluated and the data were collected on the different characters i.e., plant height, ear height, leaf rolling, days to $50 \%$ anthesis, days to $50 \%$ silking, and grain yield (g/plot). When half of the plants had shed days to anthesis were recorded pollen, as well the days to silking when $50 \%$ of the plants had silks. At physiological maturity, plant height was measured on five guarded plants per plot and then all plants were harvested, and grain yield was measured. Grain weights were adjusted to $15.5 \%$ moisture content. All data were subjected to statistical analysis using SAS 9.2 (SAS Institute, Cary, NC, USA), according to Gomez and Gomez, 1984. The grain yield was used to assess the heat susceptibility index (HSI) according to Fischer and Maurer, 1978.

The morphological data of the two most tolerant $S_{2}$ lines (L6 and L40), the two highest yield production $\mathrm{S}_{2}$ lines (L27 and L46) under normal conditions and the worst $\mathrm{S}_{2}$ line (L32, as a negative control) under normal and stressed conditions were standardized, then the genetic similarity and the Euclidean 
distance were calculated among them. Clustering analysis was done using Minitab 18 (Minitab Ltd., Coventry, UK).

\subsection{Molecular analysis}

The molecular analyses were applied at the Department of Genetics, Faculty of Agriculture, New Valley University, Egypt.

\section{DNA isolation}

DNA isolation kit (Favorgenv Biotech Corp. Cat. No. FAPGK001) was used to extract genomic DNA from young leaves of each $\mathrm{S}_{2}$ inbred line. The extraction procedures were performed as mentioned in the manufacturer manual. DNAs were quantified and

qualified by spectrophotometer and then were adjusted to a final concentration of $50 \mathrm{ng} / \mu 1$.

\subsubsection{PCR amplification procedures}

The reactions of ISSR and SRAP markers were applied in a total volume of $25 \mu$ containing: green PCR Master Mix (12.5 $\mu$ l) including 2X (4 dNTPs mixture $(400 \mu \mathrm{M}), 50$ units/ml Taq DNA polymerase, and $3 \mathrm{mM} \mathrm{MgCl} 2), 2 \mu \mathrm{l}$ of ISSR primer $(10 \mu \mathrm{M})$ or 1 $\mu \mathrm{l}$ for each forward and reverse SRAP primers (10 $\mu \mathrm{M}$ ) and $2 \mu \mathrm{l}$ of DNA (50 ng) and $8.5 \mu \mathrm{l}$ of $\mathrm{ddH}_{2} \mathrm{O}$. The amplifications were applied in a thermal cycler (Labocon, U.K.) PCR. Nine ISSR and seven SRAP primers (by metabion) were applied (Table 1).

Table 1. Names and sequences of the ISSR and SRAP primers used in this study.

\begin{tabular}{|c|c|c|c|c|}
\hline ISSR primers & Sequence (5' to $\left.3^{\prime}\right)$ & & SRAP primers & Sequence (5' to $\left.3^{\prime}\right)$ \\
\hline UBC 807 & $(\mathrm{AG}) 8 \mathrm{~T}$ & 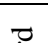 & Me 1 & TGAGTCCAAACCGGATA \\
\hline UBC 808 & $(\mathrm{AG}) 8 \mathrm{C}$ & $\Xi$ & Me 4 & TGAGTCCAAACCGGACC \\
\hline UBC 810 & (GA)8T & 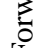 & Me 5 & TGAGTCCAAACCGGAAG \\
\hline UBC 811 & (GA)8C & & Me 8 & TGAGTCCTTTCCGGTGC \\
\hline UBC 812 & (GA) $8 \mathrm{~A}$ & & Em 1 & GACTGCGTACGAATTAAT \\
\hline UBC 816 & (CA) $8 \mathrm{~T}$ & $\ddot{\infty}$ & Em 2 & GACTGCGTACGAATTTGC \\
\hline UBC 817 & (CA)8A & $\stackrel{\bar{D}}{D}$ & Em 3 & GACTGCGTACGAATTGAC \\
\hline UBC 818 & (CA) $8 \mathrm{G}$ & $\mathscr{2}$ & Em 8 & GACTGCGTACGAATTCTG \\
\hline UBC 823 & (TC) $8 \mathrm{C}$ & & E m10 & GACTGCGTACGAATTCAG \\
\hline
\end{tabular}

The PCR program for ISSR marker started with initial denaturation for $5 \mathrm{~min}$ at $94^{\circ} \mathrm{C}$, then 38 cycles were applied including denaturation at $94^{\circ} \mathrm{C}$ for $45 \mathrm{sec}$, annealing for $1 \mathrm{~min}$ at $48^{\circ} \mathrm{C}$, extension at $72^{\circ} \mathrm{C}$ for 2 min and a final extension at $72^{\circ} \mathrm{C}$ for $7 \mathrm{~min}$. The PCR program for SRAP marker was managed as following: initial denaturation for $5 \mathrm{~min}$ at $94^{\circ} \mathrm{C}$, five cycles included three steps: denaturation for $1 \mathrm{~min}$ at $94^{\circ} \mathrm{C}, 1 \mathrm{~min}$ annealing at $35^{\circ} \mathrm{C}$ and elongation for 1 min at $72{ }^{\circ} \mathrm{C}$, then followed by 35 cycles $(1 \mathrm{~min}$ at $94^{\circ} \mathrm{C}, 1 \mathrm{~min}$ annealing at $50^{\circ} \mathrm{C}$ and 2 min elongation at $72^{\circ} \mathrm{C}$ ) and a final extension at $72^{\circ} \mathrm{C}$ for $7 \mathrm{~min}$. The products of PCR amplifications were separated on $1.5 \%$ and $2.5 \%$ agarose gels for ISSR and SRAP, respectively. The separations were performed in $1 \times \mathrm{TBE}$ (Tris-Borate-EDTA) ruining buffer at 5 Volt $/ \mathrm{cm}$.

\section{Data analysis}

The present and absent bands were scored as 1 and 0 , respectively. Dendrograms were constructed using the unweighted pair group method with arithmetic average (UPGMA) based on similarity matrix data (Jaccard, 1908). The computational package MVSP 3.1. software program was applied to assess the cluster analysis. Some indices were calculated (polymorphic information contents (PIC, Anderson et al., 1993), resolving power (Rp, Prevost and Wilkinson, 1999) and marker index (MI, Powell et al., 1996). To calculate the correlation among all the used markers for evaluating the 5 selected $\mathrm{S}_{2}$ inbred lines (morphological traits under normal and stressed conditions, ISSR, SRAP and combined), the correspondence-coefficient was assessed based on their genetic similarity matrices by using the Mantel test (Mantel, 1967).

\section{Results and Discussion}

\subsection{Mean performance of inbred lines}

The results in Table 2, show significant differences among the $60 \mathrm{~S}_{1}$ and $\mathrm{S}_{2}$ inbred lines for the traits, plant height, ear height, leaf rolling, days to $50 \%$ anthesis, days to $50 \%$ silking and grain yield (g/plot) under normal and heat stress conditions. except the plant height was not significant under heat stress (Tables 2 and 3 , respectively). The mean performances for the evaluated traits of the $S_{1}$ and $S_{2}$ inbred lines are displayed in Tables 2 and 3 , respectively. Concerning the plant height, the mean values of $S_{1}$ lines under normal conditions were ranged from $75.00 \mathrm{~cm}$ for L35 to $213.50 \mathrm{~cm}$ for L42 line with an average of $138.00 \mathrm{~cm}$. These values in $\mathrm{S}_{2}$ generation ranged from $103 \mathrm{~cm}$ for L33 to $185 \mathrm{~cm}$ for L42. On the other hand, under high temperature the values of $S_{1}$ lines ranged 
from 120.00 to $165 \mathrm{~cm}$ for the L4 and L38, respectively, with an average of $146.59 \mathrm{~cm}$. Moreover, the values of the $S_{2}$ lines ranged from 117.67 to $177.67 \mathrm{~cm}$ for L4 and L46, respectively. Plant height varied among the $S_{1}$ and as well among $\mathrm{S}_{2}$ inbred lines in both normal and the stress conditions according to their genetic makeup, this is consistent with many previous studies (Chen et al., 2012; Surender et al., 2017; Tripathi et al., 2019). These variations were more significant under normal conditions, but their average was higher under heat stress conditions, indicating the exposed temperature during stress time was more optimal for vegetative growth than during normal time. For the ear height, the mean values significantly varied among the inbred lines in both generations under both conditions. Under normal conditions, the values of $S_{1}$ lines ranged from $33.50 \mathrm{~cm}$ for L35 to $86.50 \mathrm{~cm}$ for L42 with an average of $64.46 \mathrm{~cm}$. Among the $S_{2}$ lines, the L42 had the highest ear height $(82.88 \mathrm{~cm})$, while L32 had the lowest value $(47.00 \mathrm{~cm})$. Whereas, under heat stress conditions the values of $S_{1}$ lines ranged from 49.50 for L32 to $90 \mathrm{~cm}$ for L59 with an average of $74.83 \mathrm{~cm}$. In $\mathrm{S}_{2}$ generation these values ranged from $57.90 \mathrm{~cm}$ for L4 to $86.70 \mathrm{~cm}$ for L6. Like the plant height, the ear height trait was not affected by the heat stress due to may be the heat stress planting date was not limited to plant growth, this ties well with the previous findings that showed a positive correlation between the two traits (Tripathi et al., 2019). Moreover, increasing the ear height leads to more photosynthates loss from the leaves which affect the crop yield. Thus, this trait is one of the potential traits in improving maize yield (Khayatnezhad et al., 2010). For the leaf rolling, the performance of the $S_{1}$ inbred lines under normal conditions varied from 1.50 for L9 to 5.00 for L49, while under heat stress ranged from 1.50 for L15 and L27 to 5.00 for L3, L45, L49 and L55. However, in the $S_{2}$ generation the values under normal conditions ranged from 1.20 to 3.30 for L46 and L20, respectively. Under stress conditions, the L32 had the highest value (4.33), while L27 had the lowest value (1.50). On the other hand, days to $50 \%$ anthesis differed significantly among the evaluated inbred lines at the two conditions. Under the normal conditions, the values of $S_{1}$ inbreed lines ranged from 63.00 days for L15 to 79.00 days for L37 with an average of 72.19 days. Moreover, in the $S_{2}$ generation, L40 had the shortest period (64 days), but
L59 had the longest period (76 days). Under high temperature stress, in the $S_{1}$ generation the $\mathrm{L} 9$ had the shortest period (63.5 days), while the L39 had the longest period (77 days) for $50 \%$ anthesis. In the $\mathrm{S}_{2}$ generation the shortest period (64.65 days) was recorded by L14, while the longest period (76.50 days) was recorded by L7. Regarding the days to $50 \%$ silking, in the $\mathrm{S}_{1}$ generation, the L15 was the earliest line (70 days) under normal conditions, while under stress temperature was L42 (73 days). On the other hand, L3, L37 and L58 were the latest (86 days) lines under normal conditions, while under heat stress were L18 and L39 (85 days) $S_{1}$ inbreed lines. Moreover, in the $S_{2}$ generation L16 (69 days) and L6 (73.80 days) were the earliest under the normal and stressed conditions, respectively while, L59 (83.67 days) and L32 (85.90 days) were the latest lines under the normal and stressed conditions, respectively. Under the heat stress conditions, the anthesis silking interval increased causing a reduction in grain yield. However, the most tolerant genotypes had the shortest anthesis siliking interval. Thus, shorter anthesis siliking interval is a better characteristic to determine more tolerant genotypes. These results corroborate the earlier findings of Kandel et al. (2017). The grain yield trait significantly fluctuated among all the $S_{1}$ or $S_{2}$ inbred lines under the normal and the stress conditions. Under the favorable conditions, the values of $S_{1}$ inbred lines varied from 169.00 to $975.00 \mathrm{~g}$ for L32 and L27, respectively. In $\mathrm{S}_{2}$ generation these values ranged from 199.77 to $980.22 \mathrm{~g}$ for also L32 and L27, respectively. The heat stress conditions highly reduced the grain yield of all the $\mathrm{S}_{1}$ inbred lines. The reduction varied from $25.83 \%$ for L40 to 100\% for 23 lines. On the other hand, the L6 line had the highest grain yield value (396.50 g). Also, the yield of $S_{2}$ inbred lines was affected by the heat stress conditions and the L6 line had the highest grain yield value $(560.45 \mathrm{~g})$. The reduction in grain yield may be due to that the temperature above $38^{\circ} \mathrm{C}$ during grain filling may affect the photosynthetic rate, pollen viability and fertilization (Rowhani et al., 2011; TAO et al., 2016).

\subsection{Heat stress tolerance assessment}

The heat susceptible index (HSI) was estimated to discriminate among the $S_{1}$ or/and $S_{2}$ inbred lines for their heat stress tolerance capacity (Table 2 and 3, respectively). The estimation was done as the result of variation in yield performance between normal and 
stress environments. Based on the HSI value and according to Khanna-Chopra and Viswanathan (1999), all the $\mathrm{S}_{1}$ lines were classified into three groups, one of them $(\mathrm{H})$ contained the highest heat stress-tolerant lines (2 lines: L6 and L40) which had HSI value less than 0.5 . The second group (M) included the most moderate heat-stress tolerant lines (27 lines) which had an HSI values from 0.5 to less than 1 . The third group (L) gathered the least heatstress tolerant lines (31 lines) which had HSI values equal to or more than 1. Moreover, as well as the $S_{2}$ lines were also divided into three groups, the highest heat stress-tolerant group contained L6 and L40, the moderate heat-stress tolerant group included 24 lines and the heat-stress sensitive group contained L7, L16, L32 and L33.

Table 2. Mean performance of the $60 \mathrm{~S}_{1}$ inbred lines for the studied traits under normal $(\mathrm{N})$ and heat stress environments (HS), heat susceptibility index (HSI) and the tolerance (Tol).

\begin{tabular}{|c|c|c|c|c|c|c|c|c|c|c|c|c|c|c|}
\hline \multirow[t]{2}{*}{$\mathrm{S}_{1}$ lines } & \multicolumn{2}{|c|}{ Plant height $(\mathrm{cm})$} & \multicolumn{2}{|c|}{ Ear height $(\mathrm{cm})$} & \multicolumn{2}{|c|}{ Leaf rolling } & \multicolumn{2}{|c|}{$\begin{array}{c}\text { Days to } 50 \% \\
\text { anthesis }\end{array}$} & \multicolumn{2}{|c|}{$\begin{array}{l}\text { Days to } 50 \% \\
\text { silking }\end{array}$} & \multicolumn{2}{|c|}{ Grain yield (g/plot) } & \multirow[t]{2}{*}{ HSI } & \multirow[t]{2}{*}{ Tol } \\
\hline & $\mathrm{N}$ & HS & $\mathrm{N}$ & HS & $\mathrm{N}$ & HS & $\mathrm{N}$ & HS & $\mathrm{N}$ & HS & $\mathrm{N}$ & HS & & \\
\hline $\begin{array}{l}\text { L1 } \\
\end{array}$ & 130.50 & 146.00 & 61.00 & 74.20 & 3.500 & 3.50 & 71.00 & 71.00 & 79.00 & 79.00 & 271.00 & 86.00 & 0.86 & $M$ \\
\hline L2 & 126.00 & 145.00 & 60.50 & 74.50 & 3.50 & 4.50 & 76.00 & 73.00 & 83.00 & 84.00 & 384.00 & 0.00 & 1.27 & $\mathrm{~L}$ \\
\hline L3 & 86.50 & 152.00 & 43.50 & 84.50 & 2.50 & 5.00 & 78.00 & 70.00 & 86.00 & 80.00 & 542.00 & 0.00 & 1.27 & $\mathrm{~L}$ \\
\hline L4 & 145.00 & 120.00 & 77.50 & 60.50 & 4.00 & 3.50 & 73.50 & 71.00 & 81.00 & 78.00 & 409.00 & 125.50 & 0.88 & $\mathrm{M}$ \\
\hline L5 & 125.00 & 162.50 & 55.00 & 87.00 & 2.50 & 3.00 & 75.50 & 69.00 & 84.00 & 76.50 & 215.00 & 95.00 & 0.71 & M \\
\hline L6 & 136.50 & 164.50 & 69.00 & 87.10 & 2.00 & 2.00 & 71.50 & 69.50 & 79.00 & 74.00 & 638.00 & 396.50 & 0.48 & $\mathrm{H}$ \\
\hline L7 & 126.00 & 150.50 & 57.50 & 82.50 & 2.50 & 4.00 & 73.50 & 74.50 & 81.00 & 82.50 & 421.00 & 123.00 & 0.90 & $\mathrm{M}$ \\
\hline L8 & 157.50 & 162.50 & 77.00 & 78.00 & 4.00 & 3.50 & 72.50 & 73.00 & 80.50 & 80.00 & 225.50 & 86.50 & 0.78 & M \\
\hline L9 & 103.00 & 140.00 & 53.50 & 66.00 & 1.50 & 4.00 & 73.50 & 63.50 & 81.00 & 75.50 & 535.00 & 0.00 & 1.27 & $\mathrm{~L}$ \\
\hline L10 & 140.50 & 163.00 & 62.00 & 72.50 & 3.00 & 4.50 & 69.00 & 70.50 & 76.50 & 80.50 & 276.00 & 0.00 & 1.27 & $\mathrm{~L}$ \\
\hline L11 & 150.50 & 161.85 & 68.00 & 76.00 & 4.50 & 3.50 & 74.50 & 76.00 & 82.50 & 83.00 & 245.50 & 129.50 & 0.60 & M \\
\hline L12 & 145.00 & 153.50 & 65.00 & 68.90 & 4.00 & 3.50 & 72.00 & 73.00 & 84.50 & 80.50 & 227.00 & 116.50 & 0.62 & M \\
\hline L13 & 159.00 & 135.50 & 67.50 & 71.00 & 3.00 & 4.00 & 67.50 & 72.00 & 75.00 & 79.00 & 387.50 & 141.50 & 0.80 & M \\
\hline L14 & 169.00 & 162.00 & 80.50 & 84.50 & 2.00 & 3.50 & 70.00 & 72.00 & 83.00 & 79.00 & 260.50 & 127.00 & 0.65 & M \\
\hline L15 & 168.50 & 155.00 & 71.00 & 71.00 & 3.50 & 1.50 & 63.00 & 71.50 & 70.00 & 80.00 & 607.50 & 94.00 & 1.07 & $\mathrm{~L}$ \\
\hline L16 & 129.50 & 150.50 & 69.00 & 73.00 & 3.50 & 4.00 & 64.00 & 72.00 & 76.00 & 79.00 & 435.00 & 105.00 & 0.96 & M \\
\hline L17 & 150.00 & 144.50 & 63.50 & 64.30 & 2.50 & 2.50 & 68.00 & 72.00 & 76.00 & 79.00 & 398.50 & 221.50 & 0.56 & M \\
\hline L18 & 127.00 & 135.50 & 57.00 & 70.00 & 3.00 & 3.00 & 70.50 & 75.50 & 78.00 & 85.00 & 407.00 & 78.50 & 1.02 & $\mathrm{~L}$ \\
\hline L19 & 161.00 & 144.50 & 86.00 & 80.50 & 2.50 & 3.00 & 67.50 & 70.50 & 74.50 & 77.50 & 353.00 & 206.50 & 0.53 & M \\
\hline L20 & 160.50 & 156.00 & 82.50 & 85.00 & 4.00 & 3.50 & 70.50 & 73.00 & 78.00 & 80.00 & 205.00 & 106.00 & 0.61 & M \\
\hline L21 & 129.00 & 155.00 & 63.00 & 69.00 & 4.00 & 3.50 & 71.00 & 74.50 & 79.00 & 81.50 & 306.00 & 145.00 & 0.67 & M \\
\hline L22 & 141.00 & 155.00 & 66.00 & 64.00 & 3.50 & 2.50 & 72.50 & 69.50 & 79.50 & 76.50 & 301.50 & 94.50 & 0.87 & M \\
\hline L23 & 141.50 & 145.00 & 71.00 & 73.50 & 2.00 & 3.50 & 72.50 & 73.50 & 80.00 & 80.50 & 244.00 & 131.50 & 0.58 & $\mathrm{M}$ \\
\hline L24 & 128.00 & 156.00 & 60.00 & 75.00 & 4.00 & 3.00 & 73.50 & 71.50 & 81.00 & 81.50 & 598.00 & 120.50 & 1.01 & $\mathrm{~L}$ \\
\hline L25 & 132.00 & 155.00 & 57.50 & 60.00 & 3.00 & 2.50 & 66.00 & 71.00 & 73.00 & 79.50 & 715.00 & 123.00 & 1.05 & $\mathrm{~L}$ \\
\hline L26 & 150.00 & 135.00 & 70.50 & 60.00 & 3.50 & 4.50 & 73.50 & 73.00 & 81.00 & 82.50 & 304.50 & 0.00 & 1.27 & $\mathrm{~L}$ \\
\hline L27 & 169.00 & 155.00 & 85.00 & 77.50 & 2.5 & 1.5 & 69.50 & 73.00 & 77.50 & 80.00 & 975.00 & 250 & 0.94 & M \\
\hline L28 & 141.50 & 164.00 & 61.50 & 86.00 & 2.50 & 3.00 & 70.00 & 68.50 & 78.00 & 77.50 & 694.50 & 132.50 & 1.02 & $\mathrm{~L}$ \\
\hline L29 & 111.00 & 164.50 & 51.00 & 70.50 & 2.00 & 3.50 & 72.50 & 69.00 & 80.00 & 78.50 & 195.00 & 0.00 & 1.27 & $\mathrm{~L}$ \\
\hline L30 & 110.00 & 156.50 & 44.50 & 63.50 & 3.00 & 2.50 & 73.50 & 75.50 & 81.00 & 82.50 & 320.50 & 161.00 & 0.63 & M \\
\hline L31 & 141.50 & 148.50 & 55.50 & 68.00 & 3.00 & 2.00 & 75.00 & 75.50 & 83.00 & 82.50 & 243.00 & 130.50 & 0.59 & M \\
\hline L32 & 110.00 & 142.50 & 43.50 & 49.50 & 3.00 & 4.50 & 73.00 & 73.00 & 82.00 & 82.50 & 169.00 & 0.00 & 1.27 & $\mathrm{~L}$ \\
\hline L33 & 103.00 & 149.50 & 65.00 & 79.00 & 3.00 & 4.00 & 71.00 & 69.50 & 78.00 & 76.50 & 371.00 & 110.00 & 0.89 & M \\
\hline L34 & 116.50 & 145.40 & 46.00 & 69.50 & 3.00 & 3.00 & 77.00 & 72.00 & 84.50 & 77.00 & 244.00 & 93.50 & 0.78 & M \\
\hline L35 & 75.00 & 140.00 & 33.50 & 74.00 & 3.00 & 4.50 & 74.00 & 74.50 & 83.00 & 82.00 & 319.00 & 0.00 & 1.27 & $\mathrm{~L}$ \\
\hline L36 & 116.50 & 144.80 & 55.00 & 72.90 & 3.00 & 3.00 & 72.50 & 73.00 & 81.00 & 79.00 & 460.00 & 138.50 & 0.88 & M \\
\hline L37 & 162.50 & 146.85 & 61.00 & 72.35 & 2.50 & 3.00 & 79.00 & 75.50 & 86.00 & 83.50 & 351.50 & 0.00 & 1.27 & $\mathrm{~L}$ \\
\hline L38 & 131.50 & 165.00 & 70.00 & 87.00 & 3.50 & 2.50 & 68.00 & 76.50 & 76.00 & 81.50 & 605.00 & 0.00 & 1.27 & $\mathrm{~L}$ \\
\hline L39 & 130.50 & 151.65 & 72.00 & 84.50 & 3.00 & 4.50 & 75.00 & 77.00 & 83.00 & 85.00 & 417.00 & 0.00 & 1.27 & $\mathrm{~L}$ \\
\hline L40 & 140.50 & 152.00 & 68.50 & 76.40 & 2.50 & 2.50 & 70.50 & 69.50 & 78.00 & 74.00 & 317.50 & 235.50 & 0.33 & $\mathrm{H}$ \\
\hline L41 & 139.00 & 158.00 & 67.50 & 81.00 & 2.50 & 3.50 & 68.00 & 71.00 & 75.00 & 74.50 & 719.00 & 119.50 & 1.06 & $\mathrm{~L}$ \\
\hline L42 & 213.50 & 162.50 & 86.50 & 78.60 & 3.00 & 4.00 & 69.50 & 69.50 & 77.00 & 73.00 & 304.00 & 114.50 & 0.79 & M \\
\hline L43 & 152.50 & 152.00 & 66.50 & 75.50 & 4.00 & 4.50 & 73.00 & 73.00 & 80.50 & 76.50 & 391.50 & 0.00 & 1.27 & $\mathrm{~L}$ \\
\hline L44 & 156.00 & 147.50 & 86.00 & 77.35 & 3.00 & 4.00 & 66.00 & 74.00 & 73.00 & 77.50 & 420.50 & 0.00 & 1.27 & $\mathrm{~L}$ \\
\hline L45 & 162.50 & 150.50 & 70.00 & 80.50 & 4.00 & 5.00 & 72.00 & 70.50 & 79.00 & 74.00 & 238.50 & 0.00 & 1.27 & $\mathrm{~L}$ \\
\hline L46 & 194.00 & 157.50 & 67.50 & 82.60 & 3.00 & 2.00 & 71.50 & 73.50 & 78.50 & 79.50 & 936.50 & 256.50 & 0.92 & M \\
\hline L47 & 144.00 & 146.00 & 68.00 & 75.50 & 3.00 & 4.50 & 73.50 & 75.00 & 80.50 & 82.50 & 370.00 & 0.00 & 1.27 & $\mathrm{~L}$ \\
\hline L48 & 134.00 & 143.30 & 61.50 & 73.00 & 4.00 & 4.50 & 76.50 & 73.00 & 83.50 & 82.00 & 305.50 & 0.00 & 1.27 & $\mathrm{~L}$ \\
\hline L49 & 130.00 & 137.50 & 64.00 & 62.50 & 5.00 & 5.00 & 71.0 & 69.50 & 75.00 & 78.00 & 225.00 & 0.00 & 1.27 & $\mathrm{~L}$ \\
\hline L50 & 88.50 & 127.50 & 43.00 & 76.50 & 3.00 & 3.50 & 76.50 & 74.00 & 84.00 & 81.50 & 349.00 & 0.00 & 1.27 & $\mathrm{~L}$ \\
\hline L51 & 132.50 & 121.30 & 56.50 & 75.50 & 2.50 & 3.00 & 74.00 & 71.50 & 81.50 & 79.00 & 690.50 & 105.00 & 1.07 & $\mathrm{~L}$ \\
\hline L52 & 156.00 & 132.50 & 66.50 & 71.90 & 2.50 & 4.50 & 74.50 & 72.00 & 82.50 & 79.50 & 952.00 & 0.00 & 1.27 & $\mathrm{~L}$ \\
\hline L53 & 90.00 & 135.00 & 47.50 & 73.00 & 4.00 & 4.50 & 77.00 & 71.00 & 85.00 & 74.50 & 281.50 & 0.00 & 1.27 & $\mathrm{~L}$ \\
\hline L54 & 153.50 & 144.50 & 68.50 & 87.50 & 3.00 & 3.50 & 76.50 & 70.50 & 84.00 & 78.00 & 342.00 & 123.00 & 0.81 & M \\
\hline L55 & 128.00 & 123.00 & 71.00 & 80.00 & 2.00 & 5.00 & 70.00 & 74.00 & 77.50 & 82.50 & 791.50 & 0.00 & 1.27 & $\mathrm{~L}$ \\
\hline L56 & 128.50 & 122.50 & 55.00 & 73.00 & 4.50 & 3.00 & 75.00 & 72.00 & 82.50 & 79.50 & 579.50 & 0.00 & 1.27 & $\mathrm{~L}$ \\
\hline L57 & 143.50 & 120.50 & 78.50 & 74.50 & 3.50 & 3.50 & 71.50 & 69.50 & 83.50 & 75.00 & 326.00 & 145.50 & 0.70 & M \\
\hline L58 & 143.00 & 124.50 & 66.00 & 75.00 & 3.00 & 2.50 & 78.00 & 73.00 & 86.00 & 76.50 & 398.50 & 0.00 & 1.27 & $\mathrm{~L}$ \\
\hline L59 & 165.50 & 132.00 & 66.00 & 90.00 & 3.00 & 3.00 & 76.00 & 70.50 & 83.00 & 76.50 & 346.00 & 113.50 & 0.85 & M \\
\hline L60 & 148.50 & 125.5 & 85.00 & 83.00 & 4.00 & 2.50 & 70.50 & 70.00 & 77.50 & 79.00 & 618.00 & 110.50 & 1.04 & $\mathrm{~L}$ \\
\hline F value & $* *$ & $\mathrm{~ns}$ & $* *$ & $* *$ & $*$ & $* *$ & $* *$ & $* *$ & $* *$ & $*$ & $* *$ & $* *$ & - & - \\
\hline LSD 0.05 & 38.56 & 29.18 & 16.80 & 12.62 & 1.32 & 1.18 & 7.41 & 3.33 & 12.06 & 4.44 & 124.25 & 4.71 & - & - \\
\hline
\end{tabular}

*** $*$ and ${ }^{\mathrm{ns}}$ significant, highly significant and non-significant at 0.05 and 0.01 levels of probability, respectively; Capital letters $\mathrm{H}, \mathrm{M}$ and $\mathrm{L}$ refer to high, moderate and low tolerance degree, respectively. 
Table 3. Mean performance of the $30 \mathrm{~S}_{2}$ inbred lines for the studied traits under normal $(\mathrm{N})$ and heat stress environments (HS), heat susceptibility index (HSI) and the tolerance (Tol).

\begin{tabular}{|c|c|c|c|c|c|c|c|c|c|c|c|c|c|c|}
\hline \multirow[t]{2}{*}{$\mathrm{S}_{2}$ lines } & \multicolumn{2}{|c|}{ Plant height $(\mathrm{cm})$} & \multicolumn{2}{|c|}{ Ear height $(\mathrm{cm})$} & \multicolumn{2}{|c|}{ Leaf rolling } & \multicolumn{2}{|c|}{$\begin{array}{c}\text { Days to } 50 \% \\
\text { anthesis }\end{array}$} & \multicolumn{2}{|c|}{$\begin{array}{l}\text { Days to } 50 \% \\
\text { silking }\end{array}$} & \multicolumn{2}{|c|}{ Grain yield (g/plot) } & \multirow[t]{2}{*}{ HSI } & \multirow[t]{2}{*}{ Tol } \\
\hline & $\mathrm{N}$ & HS & $\mathrm{N}$ & $\mathrm{HS}$ & $\mathrm{N}$ & HS & $\mathrm{N}$ & $\mathrm{HS}$ & $\mathrm{N}$ & HS & $\mathrm{N}$ & HS & & \\
\hline L1 & 145.50 & 154.00 & 62.50 & 75.50 & 3.00 & 3.00 & 70.00 & 69.67 & 76.50 & 77.00 & 265.45 & 145.3 & 0.96 & $\mathrm{M}$ \\
\hline L4 & 150.50 & 117.67 & 67.50 & 57.90 & 3.00 & 3.00 & 69.00 & 70.00 & 74.00 & 77.00 & 399.99 & 215.67 & 0.98 & M \\
\hline L5 & 162.57 & 167.69 & 63.67 & 85.00 & 2.67 & 3.00 & 72.00 & 70.00 & 77.67 & 77.50 & 221.56 & 132.67 & 0.85 & M \\
\hline L6 & 141.67 & 161.97 & 61.00 & 86.70 & 2.00 & 2.50 & 68.67 & 68.67 & 74.67 & 73.80 & 712.72 & 560.45 & 0.45 & $\mathrm{H}$ \\
\hline L7 & 151.00 & 158.00 & 57.68 & 80.00 & 2.55 & 3.33 & 70.67 & 76.50 & 75.00 & 84.00 & 499.1 & 120.2 & 1.62 & L \\
\hline L8 & 155.00 & 160.55 & 62.66 & 73.00 & 3.00 & 3.00 & 67.50 & 75.67 & 74.00 & 84.00 & 243.67 & 133.67 & 0.96 & M \\
\hline L11 & 150.43 & 161.70 & 61.00 & 77.69 & 3.00 & 3.00 & 72.00 & 73.67 & 78.00 & 80.00 & 230.5 & 145.55 & 0.78 & M \\
\hline L12 & 145.00 & 154.60 & 60.00 & 66.00 & 3.00 & 4.00 & 68.00 & 75.00 & 73.67 & 81.67 & 215.6 & 139.9 & 0.75 & M \\
\hline L13 & 156.00 & 155.80 & 67.89 & 69.00 & 2.36 & 3.00 & 67.50 & 71.00 & 72.00 & 78.50 & 389.87 & 217.67 & 0.94 & M \\
\hline L14 & 169.00 & 172.54 & 75.46 & 86.68 & 3.00 & 3.00 & 68.67 & 64.65 & 78.67 & 82.50 & 290.87 & 165.5 & 0.92 & M \\
\hline L16 & 148.00 & 155.77 & 64.00 & 73.00 & 3.00 & 4.00 & 64.00 & 71.00 & 69.00 & 79.67 & 528.89 & 113.7 & 1.67 & $\mathrm{~L}$ \\
\hline L17 & 146.33 & 158.90 & 53.68 & 62.69 & 2.97 & 2.74 & 66.00 & 70.00 & 72.00 & 77.00 & 422.8 & 225.25 & 0.99 & M \\
\hline L19 & 161.00 & 162.67 & 64.00 & 75.67 & 2.75 & 3.00 & 67.67 & 70.67 & 73.67 & 78.50 & 368.55 & 224.67 & 0.83 & M \\
\hline L20 & 157.22 & 156.30 & 73.67 & 85.00 & 3.30 & 4.00 & 70.50 & 70.67 & 78.00 & 77.67 & 262.55 & 143.4 & 0.97 & M \\
\hline L21 & 154.00 & 165.90 & 61.00 & 71.67 & 3.00 & 3.65 & 70.00 & 74.00 & 75.33 & 81.67 & 342.7 & 185.1 & 0.98 & M \\
\hline L22 & 146.00 & 152.67 & 58.67 & 62.65 & 2.66 & 3.00 & 70.50 & 71.50 & 76.00 & 79.00 & 361.67 & 198.99 & 0.96 & M \\
\hline L23 & 146.50 & 161.70 & 56.00 & 64.89 & 2.67 & 4.00 & 68.67 & 72.50 & 74.00 & 80.67 & 234.67 & 125.45 & 0.99 & M \\
\hline L27 & 167.00 & 162.67 & 75.00 & 72.75 & 1.33 & 1.50 & 67.50 & 75.50 & 71.67 & 83.67 & 980.22 & 522.88 & 0.99 & M \\
\hline L30 & 150.00 & 156.97 & 57.67 & 63.67 & 3.00 & 2.87 & 71.00 & 75.00 & 75.00 & 82.67 & 335.66 & 178.98 & 0.99 & M \\
\hline L31 & 149.22 & 156.00 & 55.64 & 68.00 & 2.62 & 2.00 & 75.00 & 75.00 & 79.00 & 82.67 & 269.9 & 185.67 & 0.66 & M \\
\hline L32 & 139.00 & 157.69 & 47.00 & 60.00 & 2.46 & 4.33 & 70.00 & 74.50 & 76.67 & 85.90 & 199.77 & 78.88 & 1.29 & $\mathrm{~L}$ \\
\hline L33 & 103.00 & 157.67 & 65.00 & 77.00 & 3.00 & 3.50 & 71.00 & 69.00 & 78.00 & 7690 & 485.5 & 105.98 & 1.66 & $\mathrm{~L}$ \\
\hline L34 & 154.00 & 160.00 & 56.00 & 83.00 & 3.00 & 3.35 & 71.00 & 73.50 & 75.00 & 82.67 & 300.93 & 160.67 & 0.99 & M \\
\hline L36 & 148.00 & 155.00 & 55.00 & 76.66 & 3.00 & 3.00 & 72.50 & 73.00 & 81.00 & 80.00 & 435.9 & 238.67 & 0.96 & M \\
\hline L40 & 148.13 & 162.77 & 62.78 & 84.67 & 2.00 & 2.50 & 64.00 & 68.67 & 68.67 & 74.00 & 430.87 & 358.77 & 0.36 & $\mathrm{H}$ \\
\hline L42 & 185.00 & 168.67 & 82.88 & 78.64 & 3.00 & 3.67 & 69.00 & 69.67 & 75.00 & 74.67 & 335.9 & 188.64 & 0.93 & M \\
\hline L46 & 182.12 & 177.67 & 76.67 & 83.00 & 1.20 & 2.00 & 68.50 & 73.67 & 73.50 & 79.67 & 958.11 & 520.78 & 0.97 & M \\
\hline L54 & 151.46 & 168.64 & 68.25 & 79.98 & 3.00 & 3.00 & 73.00 & 69.50 & 78.60 & 77.00 & 365.64 & 195.74 & 0.99 & M \\
\hline L57 & 143.24 & 120.66 & 78.89 & 74.50 & 3.00 & 4.00 & 71.00 & 69.50 & 83.00 & 75.00 & 368.77 & 197.67 & 0.99 & M \\
\hline L59 & 165.15 & 152.89 & 66.60 & 80.00 & 3.00 & 3.71 & 76.00 & 70.67 & 83.67 & 76.67 & 380.12 & 203.67 & 0.99 & M \\
\hline $\mathrm{F}$ value & $* *$ & $* *$ & ** & ** & $* *$ & $* *$ & $* *$ & $* *$ & $* *$ & $* *$ & $* *$ & $* *$ & - & - \\
\hline $\begin{array}{l}\text { LSD } \\
0.05\end{array}$ & 8.41 & 5.30 & 7.65 & 6.36 & 0.68 & 2.20 & 3.13 & 2.57 & 2.74 & 0.62 & 58.57 & 27.16 & - & - \\
\hline
\end{tabular}

Heat stress affected all the inbred lines in the two generations, even some of them in the $S_{1}$ generation lost their yield ability. The most heat-stress tolerant lines had not the highest yield production under normal conditions, but they had the lowest difference between the two conditions. However, the lines had HSI value less than one in both generations indicating relative resistance to heat stress reflecting stable performance over environments. In contrast, the lines which have values greater than 1 indicating relative susceptibility to heat stress. Many researchers have used the HSI and showed that it was more efficient parameter for selecting heat tolerant with high yield genotypes (Thiry et al., 2016; Kamrani et al., 2018).

\subsection{Cluster analysis using morphological characteristics} Cluster analysis was performed for the morphological traits data of the evaluated $S_{2}$ generation to discriminate

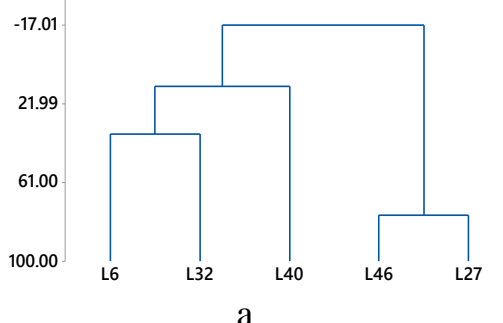

among the two most tolerant lines (L6 and L40) that have HIS values less than 0.5 , the two highest yield production lines (L27 and L46) under normal conditions and the worst line (L32, as a negative control) (Fig. 2). Under normal conditions (Fig. 2a), the five lines were classified into two main clusters, the first one contained L27 and L46. The second one was divided into two sub-clusters; one included only L40 and the other contained L6 and L32. Whereas under stress conditions (Fig. 2b), the inbred lines were distributed onto two main clusters, the first one included only L32 (the worst line), while the second one branched into two sub-clusters, one of them contained L40 and the second one divided into two groups one group gathered both L46 and L27, but the other one contained only L6.

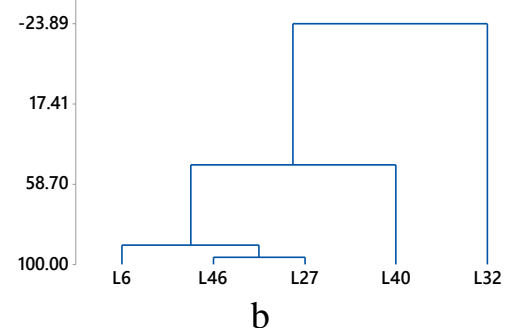

Fig.2. Clustering the 5 selected $\mathrm{S}_{2}$ inbred lines based on the studied morphological traits. a and $\mathrm{b}$ are constructed diagrams under both the normal and heat stress conditions, respectively. 


\subsection{Molecular analysis}

\subsubsection{ISSR and SRAP markers analysis}

ISSR and SRAP markers analysis were applied to verify the level of genetic variability among the five selected lines, L6, L40, L27, L46 and L32 (Figs. 3 and 4). ISSR primers amplified a total of 94 bands having sizes ranged from 145 to $1440 \mathrm{bp} .75$ bands out of them were polymorphic with a polymorphism ratio of $79.79 \%$ (Table 4 ). On the other hand, the SRAP marker detected 65 bands, 38 among them were polymorphic bands. The polymorphism ratio was $58.46 \%$. Genetic variability has successfully been determined by ISSR and SRAP markers in many plants (Liu et al., 2008; Shao et al., 2010; Liu et al., 2012; Bashandy and El-Shaieny, 2016; Mahmoud and Abd El-Fatah, 2020). The detected polymorphism differed in both markers due to each of them targeting different sequences of the genome. The value of detected polymorphism was higher in ISSR than SRAP, because the SRAP is designed to detect any variations in coding sequences that having low mutation rates and are more conserved among individuals (Liu et al., 2008). this result is consistent with the findings of Shao et al. (2010), Liu et al. (2012) and Luo et al. (2020). On the contrary, Mahmoud and Abd El-Fatah (2020) used molecular markers to evaluate the response of 16 faba bean genotypes to Fusarium wilt. Their results showed the SRAP marker had a higher polymorphism (82.53\%) than the ISSR marker (62.24\%). Moreover, the PIC value was calculated to differentiate among the two markers for their ability in polymorphism detection. This value in ISSR ranged from 0.24 to 0.4 with an average of 0.309 , but in the SRAP marker it varied from 0.12 to 0.364 with an average of 0.218 . Furthermore, the primer resolving power $(\mathrm{Rp})$ was calculated to compare among the primers of each marker for their capacity in the detection of genetic variations. The $\mathrm{Rp}$ value in the ISSR marker fluctuated from 3.2 to 9.2 for UBC 807 and UBC 811 primer, respectively. For the SRAP marker, the lowest value (1.2) was recorded by Me1/Em1 primers combination, while Me1/Em10 primers combination showed the highest value (6.00). In addition, the marker index (MI) was calculated to select the preferable marker in the detection of the variability among the studied $S_{2}$ inbred lines. The ISSR marker had a higher MI value (2.56) than SRAP (1.35) indicating that the ISSR marker provided more informative data than the SRAP marker. Similar results have been found by Luo et al. (2020).

\subsubsection{Molecular relationship assessment}

Based on ISSR and SRAP data, the level of similarity among the five $S_{2}$ inbred lines was calculated according to Jaccard's similarity coefficient (Table 5). the ISSR marker showed the highest similarity ( 0.60$)$ between L46 and L27, also between L27 and L32, while the lowest similarity (0.37) was between L6 and L32. Concerning the SRAP marker, the L6 and L40 displayed the highest similarity (0.82), but the lowest similarity (0.48) was between L46 and L32. The ISSR and SRAP combined data revealed that the L46 and L27 had the highest similarity (0.65), while the L6 and L32 had the lowest similarity (0.47). Furthermore, the dendrograms of genetic similarity using ISSR, SRAP markers and combined were constructed (Fig. 5). All the dendrograms divided the $S_{2}$ inbred lines into two main clusters. The ISSR dendrogram separated the L6 in one cluster and combined the rest $S_{2}$ inbred lines in the second cluster. The second one was divided into two sub-clusters, one of them contained only L40, while the second one was divided into two groups. The first group included only the L32, while the second one combined both the L46 and L27. Concerning the SRAP dendrogram classified only the worst line (L32) in one cluster and the second cluster was divided into two sub-clusters, one of them contained only the L27. The second sub-cluster branched into two groups, the L46 was classified into one group and the second one gathered both the L6 and L40. The combined results of both markers separated only the L32 in one cluster, while the second main cluster was divided into two sub-clusters one of them included only the L6 and the second one sub-divided into two groups. One group contained the L40, but the other group gathered both the L46 and L27.

The two markers did not construct similar dendrograms, because each of them detecting different sequences in the genome. Many researchers have applied different types of DNA markers to study the genetic variability and showed different dendrograms (Chen et al., 2013; Shahlaei et al., 2014; Bashandy et al., 2020). On the contrary, Liu et al. (2012) studied the genetic relationship among five species of Pinellia via ISSR and SRAP markers, they showed the two markers exhibited similar clustering patterns. The ISSR marker was able to isolate the line which had the highest yield under the stress conditions in one cluster, but both the SRAP and combined classified the worst line in one cluster as it also observed in the dendrogram of phenotypic traits under heat stress conditions. On the other hand, the combined ISSR and SRAP markers data constructed a closer similar 
dendrogram to the dendrogram of phenotypic traits under stress conditions. Moreover, the ISSR marker cannot detect all the transcription regions, while the SRAP amplifying
ORF regions, thus combining their data showed an extra result for detecting the variability among the genotypes.

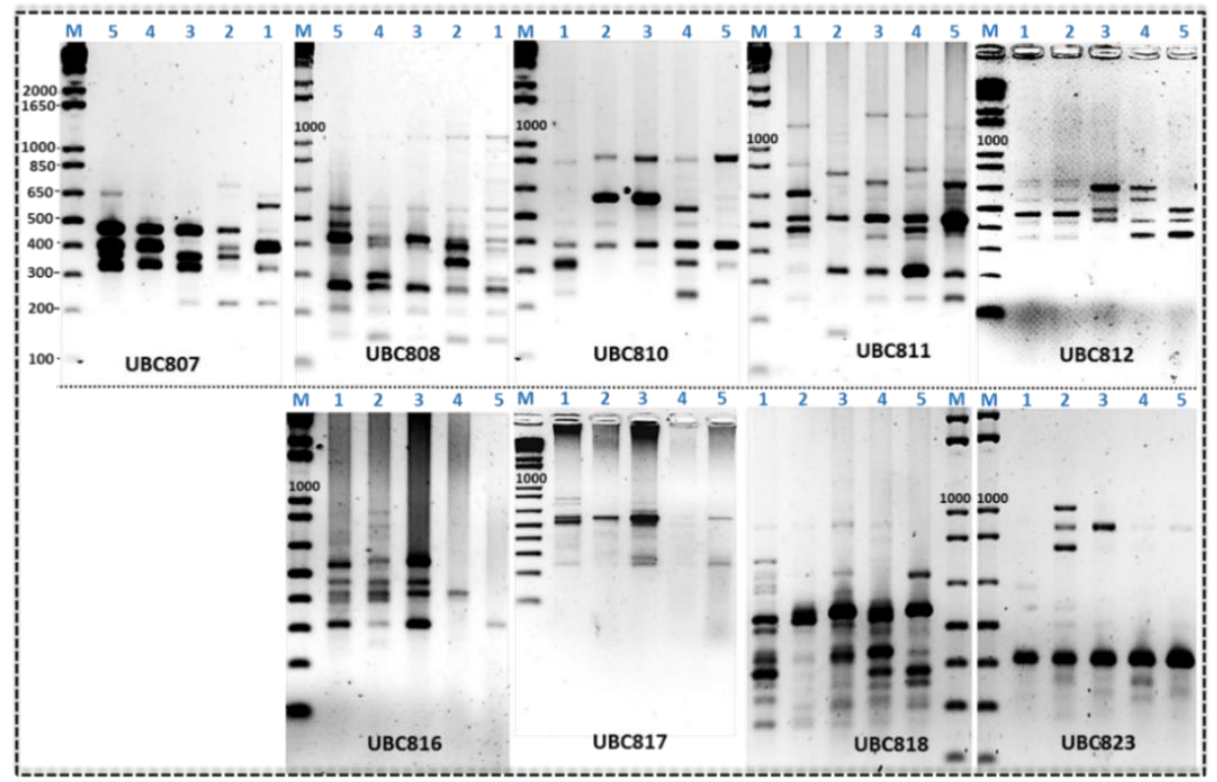

Fig.3. Banding pattern of ISSR marker of the 5 selected $\mathrm{S}_{2}$ inbred lines. M, kbp DNA ladder; 1, L6; 2, L46; 3, L40; 4, L27; 5, L32 inbred line.

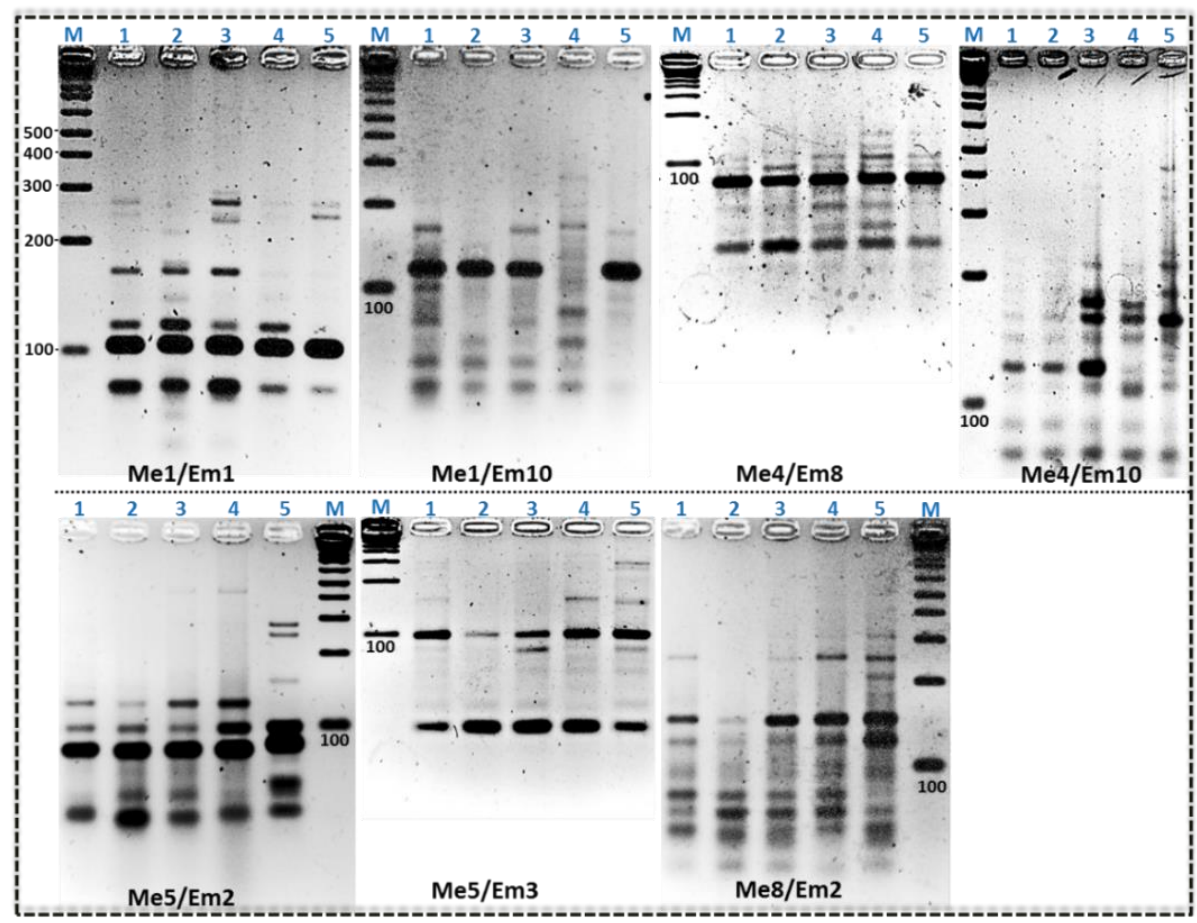

Fig.4. Banding pattern of SRAP marker of the 5 selected $S_{2}$ inbred lines. M, kbp DNA ladder; 1, L6; 2, L46; 3, L40; 4, L27; 5, L32 inbred line.

\subsection{Correlation among the used marker types}

The correlation coefficient for the similarity matrices of the used markers (morphological, ISSR, SRAP and combined (ISSR + SRAP) markers) was estimated to study the relationship among them in the evaluation of the 5 selected $\mathrm{S}_{2}$ inbred lines (Table 6). A positive correlation was detected among all the markers. Moreover, the correlation was significant and strong (0.723) and highly strong $(0.915)$ between combined and ISSR marker and morphological traits under normal conditions and SRAP marker, respectively. It was low and not significant between ISSR marker and morphological traits under heat stress and between morphological traits under normal conditions and combined $(0.015,0.270$, respectively). On the other hand, the correlation between the rest markers was moderate and not significant. The morphological traits were highly correlated with SRAP marker than 
with ISSR marker which is consistent with the finding of Shao et al. (2010). Also, they detected a poor correlation between SRAP and ISSR marker as like our results. The conflict between ISSR and SRAP in correlation with the morphological traits may be

Table 4. Polymorphism (P\%), polymorphism information content (PIC), resolving power (Rp) and marker index (MI) obtained by ISSR and SRAP markers in the 5 selected inbred lines in $\mathrm{S}_{2}$ generation.

\begin{tabular}{|c|c|c|c|c|c|c|c|c|}
\hline $\begin{array}{l}\text { Primer } \\
\text { name }\end{array}$ & $\begin{array}{c}\text { Range of } \\
\text { fragment } \\
\text { size bp } \\
\end{array}$ & $\begin{array}{l}\text { Total No. of } \\
\text { fragments }\end{array}$ & $\begin{array}{l}\text { Monomorphic } \\
\text { fragments }\end{array}$ & $\begin{array}{l}\text { Polymorphic } \\
\text { fragments }\end{array}$ & $\mathrm{P} \%$ & PIC & $\mathrm{R} \mathrm{p}$ & MI \\
\hline \multicolumn{9}{|l|}{$\overline{\text { ISSR }}$} \\
\hline UBC 807 & $210-700$ & 9 & 3 & 6 & 66.7 & 0.249 & 3.2 & 1.49 \\
\hline UBC 808 & $145-1070$ & 14 & 5 & 9 & 64.3 & 0.24 & 4.8 & 2.16 \\
\hline UBC 810 & $230-850$ & 9 & 1 & 8 & 88.9 & 0.284 & 3.6 & 2.27 \\
\hline UBC 811 & $168-1440$ & 18 & 3 & 15 & 83.3 & 0.338 & 9.2 & 5.07 \\
\hline UBC 812 & $355-701$ & 7 & 1 & 6 & 85.7 & 0.343 & 3.6 & 2.06 \\
\hline UBC 816 & $310-560$ & 6 & 0 & 6 & 100 & 0.4 & 3.6 & 2.4 \\
\hline UBC 817 & $240-917$ & 9 & 2 & 7 & 77.8 & 0.284 & 3.6 & 1.99 \\
\hline UBC 818 & $250-900$ & 14 & 3 & 11 & 78.6 & 0.263 & 4.8 & 2.89 \\
\hline UBC 823 & $325-1015$ & 8 & 1 & 7 & 87.5 & 0.38 & 4.4 & 2.66 \\
\hline Total & - & 94 & 19 & 75 & - & - & - & - \\
\hline Average & - & - & - & - & - & 0.309 & - & 2.56 \\
\hline \multicolumn{9}{|l|}{ SRAP } \\
\hline Me1/Em1 & $75-270$ & 8 & 5 & 3 & 37.5 & 0.12 & 1.2 & 0.36 \\
\hline $\mathrm{Me} 1 / \mathrm{Em} 10$ & $45-262$ & 11 & 1 & 10 & 90.9 & 0.364 & 6 & 3.64 \\
\hline Me4/Em8 & $31-154$ & 8 & 3 & 5 & 62.5 & 0.2 & 2 & 1 \\
\hline $\mathrm{Me} 4 / \mathrm{Em} 10$ & $77-425$ & 12 & 8 & 4 & 33.3 & 0.133 & 2.4 & 0.53 \\
\hline $\mathrm{Me} 5 / \mathrm{Em} 2$ & $52-455$ & 10 & 2 & 8 & 80 & 0.272 & 3.6 & 2.18 \\
\hline $\mathrm{Me} 5 / \mathrm{Em} 3$ & $30-307$ & 7 & 3 & 4 & 57.1 & 0.274 & 3.2 & 1.1 \\
\hline Me8/Em2 & $65-310$ & 9 & 5 & 4 & 44.4 & 0.16 & 2 & 0.64 \\
\hline Total & - & 65 & 27 & 38 & - & - & - & - \\
\hline Average & - & - & - & - & - & 0.218 & - & 1.35 \\
\hline
\end{tabular}

Table 5. The similarity index among the 5 selected inbred lines in $\mathrm{S}_{2}$ generation based on ISSR, SRAP and combined.

\begin{tabular}{|c|c|c|c|c|c|}
\hline $\begin{array}{c}\text { Genot } \\
\text { ypes }\end{array}$ & L6 & L46 & L40 & L27 & $\begin{array}{c}\text { Marker } \\
\text { type }\end{array}$ \\
\hline \multirow{3}{*}{ L46 } & 0.49 & & & & ISSR \\
\hline & 0.75 & & & & SRAP \\
\hline & 0.59 & & & & Combined \\
\hline \multirow{3}{*}{ L40 } & 0.40 & 0.54 & & & ISSR \\
\hline & 0.82 & 0.73 & & & SRAP \\
\hline & 0.56 & 0.61 & & & Combined \\
\hline \multirow{3}{*}{ L27 } & 0.46 & 0.60 & 0.54 & & ISSR \\
\hline & 0.70 & 0.71 & 0.75 & & SRAP \\
\hline & 0.56 & 0.65 & 0.63 & & Combined \\
\hline \multirow{3}{*}{ L32 } & 0.37 & 0.52 & 0.55 & 0.60 & ISSR \\
\hline & 0.62 & 0.48 & 0.63 & 0.54 & SRAP \\
\hline & 0.47 & 0.50 & 0.59 & 0.57 & Combined \\
\hline
\end{tabular}

\section{Conclusion}

In the current study, some derived inbred lines were evaluated in $S_{1}$ and $S_{2}$ generations for their performance to tolerate heat stress. The evaluation because they detect different DNA sequences. ISSR marker aimed to micro-satellite regions, while SRAP amplifies the open reading frames region. included some agro-morphological traits and DNA molecular marker analysis. Their response to heat stress was significantly varied among them. The L6 inbred line had the highest yield under the stress conditions in $S_{1}$ and $S_{2}$ generations. Moreover, heat susceptibility index measurement identified both L6 and L40 as the most heat stress-tolerant inbred lines. Furthermore, ISSR and SRAP molecular analysis were efficient tools in the detection of genetic variability among them. However, The L6 and L40 inbred lines can be used in breeding program in developing new heat-tolerant maize varieties.

\section{Acknowledgements}

We are grateful to the Faculty of Agriculture, New Valley University, Egypt for the financial support of this work.

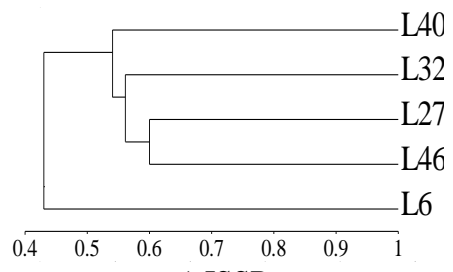

a) ISSR

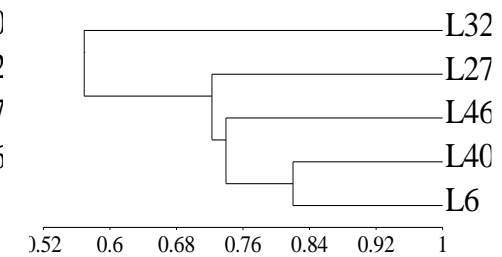

b) SRAP

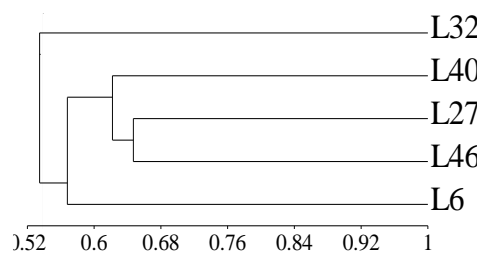

c) Combined

Fig.5. Jaccard's similarity dendrograms constructed by UPGMA based on ISSR, SRAP and combined 
Table 6. Correlation analysis among all the used markers.

\begin{tabular}{|c|c|c|c|c|}
\hline Marker type & $\begin{array}{c}\text { Morphological traits under } \\
\text { normal conditions }\end{array}$ & $\begin{array}{c}\text { Morphological traits } \\
\text { under heat stress }\end{array}$ & $\begin{array}{c}\text { ISSR } \\
\text { marker }\end{array}$ & $\begin{array}{l}\text { SRAP } \\
\text { marker }\end{array}$ \\
\hline Morphological traits under heat stress & $0.702^{\text {ns }}$ & & & \\
\hline ISSR marker & $0.420^{\mathrm{ns}}$ & $0.015^{\mathrm{ns}}$ & & \\
\hline SRAP marker & $0.915^{*}$ & $0.717^{\text {ns }}$ & $0.383^{\mathrm{ns}}$ & \\
\hline Combined (ISSR + SRAP) & $0.270^{\mathrm{ns}}$ & $0.543^{\mathrm{ns}}$ & $0.723^{*}$ & $0.336^{\mathrm{ns}}$ \\
\hline
\end{tabular}

\section{References}

Anderson, J., Churchill, G., Autrique, J., Tanksley, S., and Sorrells, M. (1993) 'Optimizing parental selection for genetic linkage maps', Genome, 36(1), pp. 181-186.

Bashandy, T. and EL-Shaieny, A. (2016) 'Screening of Cowpea (Vigna unguiculata $\mathrm{L}$. Walp) genotypes for salinity tolerance using field evaluation and molecular analysis', Journal of Agricultural Chemistry and Biotechnology, 7(9), pp. 249-255.

Bashandy, T., Hussein, A., Solma, M., Kassab, A., and Hamdon, H. (2020) 'molecular evaluation of three populations of Farafra sheep in comparison to Ossimi and Rahmani sheep breeds', Acta Universitatis Agriculturae et Silviculturae Mendelianae, 68(6), pp. 929936.

Chen, J., X.u., W., Velten, J., Xin, Z., and Stout, J. (2012) 'Characterization of maize inbred lines for drought and heat tolerance', Journal of soil and water conservation, 67(5), pp. 354-364.

Chen, S.Y., Dai, T.X., Chang, Y.T., Wang, S.S., Ou, S.L., and Chuang, W.L., et al. (2013) 'Genetic diversity among 'Ocimum'species based on ISSR, RAPD and SRAP markers', Australian Journal of Crop Science, 7(10), pp. 1463-1471.

FAO, FAOSTAT (2019). Food and agriculture organization of the United Nations, Rome, Italy.

Fischer, R., and Maurer, R. (1978) 'drought resistance in spring wheat (Triticum aestivum L.) cultivars. i. grain yield response', Australian Journal of Agricultural Research, 29(5), pp. 897-912.

Gomez, K.A., and Gomez, A.A. (1984). Statistical procedures for agriculture research. Wiley Inter Science Publication. John Wiley \& Son, Inc. New York, USA.

Jaccard, P. (1908) 'Nouvelles recherches sur la distribution florale', Bulletin de la Société vaudoise des sciences naturelles, 44, pp. 223270.
Kamrani, M., Hoseini, Y., and Ebadollahi, A. (2018) 'Evaluation for heat stress tolerance in durum wheat genotypes using stress tolerance indices', Archives of Agronomy and Soil Science, 64(1), pp. 38-45.

Kandel, M., Ghimire, S.K., Ojha, B.R., and Shrestha, J. (2017) 'Analysis of genetic diversity among the maize inbred lines (Zea mays L.) under heat stress condition', Journal of Maize Research and Development, 3(1), pp 86-97.

Khanna-Chopra, R., and Viswanathan, C. (1999) 'Evaluation of heat stress tolerance in irrigated environment of $T$. aestivum and related species. I. Stability in yield and yield components', Euphytica, 106(2), pp. 169-180.

Khayatnezhad, M., Gholamin, R., JamaatieSomarin, S., and Zabihie-Mahmoodabad, R. (2010) 'Correlation coefficient analysis between grain yield and its components in corn (Zea mays L.) hybrids', American-Eurasian Journal of Agriculture and Environmental Science, 9(1), pp 105-108.

Li, G., and Quiros, C.F. (2001) 'Sequence-related amplified polymorphism (SRAP), a new marker system based on a simple PCR reaction: its application to mapping and gene tagging in Brassica', Theoretical and applied genetics, 103(2), pp 455-461.

Liu, L., Yang, Z., Wei, S., Ouyang, Z., Wu, K., and Peng Z. (2012) 'ISSR and SRAP markers in the genetic relationship analysis among Pinellia in China', Journal of Medicinal Plants Research, 6(19), pp 3596-3602.

Liu, L.W., Zhao, L.P., Gong, Y.Q., Wang, M.X., Chen, L.M., Yang, J.L., Wang, Y., Yu, F.M., and Wang, L.Z. (2008) 'DNA fingerprinting and genetic diversity analysis of late-bolting radish cultivars with RAPD, ISSR and SRAP markers', Scientia Horticulturae, 116(3), pp 240-247.

Luo, Y., Zhang, X., Xu, J., Zheng, Y., Pu, S., Duan, Z., and Wang, Z. (2020) 'Phenotypic and molecular marker analysis uncovers the genetic diversity of the grass 
Stenotaphrumsecundatum', BMC genetics, 21(1), pp 1-12.

Mahmoud, A.F., and AbdEl-Fatah, B.E. (2020) 'Genetic diversity studies and identification of molecular and biochemical markers associated with fusarium wilt resistance in cultivated faba bean (Vicia faba)', The plant pathology journal, 36(1), pp 11-28.

Mantel, N. (1967) 'The detection of disease clustering and a generalized regression approach', Cancer research, 27(2), pp 209220.

Osti, N.P. (2019) 'Animal feed resources and their management in Nepal', Acta Scientific Agriculture, 2(10), pp 10-13.

Powell, W., Morgante, M., Andre, C., Hanafey, M., Vogel, J., and Tingey, S et al. (1996) 'The comparison of RFLP, RAPD, AFLP and SSR (microsatellite) markers for germplasm analysis', Molecular Breeding, 2(3), pp 225238.

Prasad, B., Babar, M.A., Xu, X.Y., Bai, G.H., and Klatt, A.R. (2009) 'Genetic diversity in the US hard red winter wheat cultivars as revealed by microsatellite markers', Crop and Pasture Science, 60(1), pp 16-24.

Prevost, A., and Wilkinson, M. (1999) 'A new system of comparing PCR primers applied to ISSR fingerprinting of potato cultivars', Theoretical and applied Genetics, 98(1), pp 107-112.

Rowhani, P., Lobell, D.B., Linderman, M., and Ramankutty, N. (2011) 'Climate variability and crop production in Tanzania', Agricultural and Forest Meteorology, 151(4), pp 449-460.

Sánchez, B., Rasmussen, A., and Porter, J.R. (2014) 'Temperatures and the growth and development of maize and rice: A review', Global change biology, 20(2), pp. 408-417.

Schauberger, B., Archontoulis, S., Arneth, A., Balkovic, J., Ciais, P., Deryng, D., Elliott, J., Folberth, C., Khabarov, N., and Müller, C. (2017) 'Consistent negative response of US crops to high temperatures in observations and crop models', Nature communications, $8(1)$, pp. 1-9.

Shahlaei, A., Torabi, S., and Khosroshahli, M. (2014) 'Efficacy of SCoT and ISSR markers in assessment of tomato (Lycopersicum esculentum Mill.) genetic diversity', International Journal of Biosciences, 5(2), pp 14-22.

Shao., Q.S., Guo, Q.S., Deng, Y.M., and Guo, H.P. (2010) 'A comparative analysis of genetic diversity in medicinal Chrysanthemum morifolium based on morphology, ISSR and SRAP markers', Biochemical Systematics and Ecology, 38(6), pp 1160-1169.

Surender, M., Sagare, D., Setti, P., Rani, C.V.D., Jabeen, F., Sudarshan, M.R., and Reddy, S.S. (2017) 'Mean performance of normal and QPM Maize genotypes for yield and tryptophan Content', International Journal of Current Microbiology and Applied Sciences, 6(11), pp 830-844.

Tao, Z.Q., Chen, Y.Q., Chao, L.I., Zou, J.X., Peng, Y.A.N., Yuan, S.F., and Peng, S.U.I. (2016) 'The causes and impacts for heat stress in spring maize during grain filling in the North China Plain-A review', Journal of Integrative Agriculture, 15(12), pp 2677-2687.

Thiry, A.A., Chavez, Dulanto, P.N., Reynolds, M.P., and Davies, W.J. (2016) 'How can we improve crop genotypes to increase stress resilience and productivity in a future climate? A new crop screening method based on productivity and resistance to abiotic stress', Journal of experimental botany, 67(19), pp 5593-5603.

Tripathi, M.P., Ghimire, S.K., Nair, S.K., Sah, S.K., Pandey, M.P., Vinayan, M.T., and Zaidi, P.H. (2019) 'Evaluation of heat tolerant maize (Zea mays L.) inbred lines under natural field conditions in India', Journal of Agriculture and Forestry University, 3, pp 99-104.

Zietkiewicz, E., Rafalski, A., and Labuda, D. (1994) 'Genome fingerprinting by simple sequence repeat (SSR)-anchored polymerase chain reaction amplification', Genomics, 20(2), pp 176-183. 